\title{
Hereditary gastrointestinal polyposis: Diagnosis, genetic test and risk assessment
}

\author{
Marina De Rosa*, Francesca Duraturo, Raffaella Liccardo, Paola Izzo \\ Dipartimento di Medicina Molecolare e Biotecnologie Mediche and CEINGE Biotecnologie Avanzate, Università di Napoli Federico \\ II, Naples, Italy \\ Email: ${ }^{*}$ marina.derosa@unina.it
}

Received 23 May 2013; revised 28 June 2013; accepted 10 July 2013

Copyright (C) 2013 Marina De Rosa et al. This is an open access article distributed under the Creative Commons Attribution License, which permits unrestricted use, distribution, and reproduction in any medium, provided the original work is properly cited.

\section{ABSTRACT}

Colorectal cancer (CRC) is the second cause of cancer deaths, with over 1 million new cases estimated every year. Familial adenomatous polyposis, MUTYH-associated polyposis and hamartomatous polyposis are inherited syndromes that account for $2 \%-5 \%$ of all colon cancer. The mutated genes responsible for the vast majority of these disorders, are now known (MLH1, MSH2, MSH6, PMS2, APC, MYH, LKB1, SMAD4, BMPR1A, and PTEN) and specific mutations have been identified. Molecular caracterization of inherited CRCs allows pre-symptomatic diagnosis identifying at-risk individuals and improving cancer surveillance. Adenomatous polyposis includes familial adenomatous polyposis (FAP), attenuated FAP (AFAP), and MUTYH-associated polyposis (MAP). Hamartomatous polyposis comprises Peutz-Jeghers syndrome (PJS), juvenile polyposis syndrome (JPS) and "PTEN hamartoma tumour syndrome" (PHTS). MAP is an autosomal recessive condition, while all other disorders are inherited in an autosomal dominant manner. Differential dyagnosis could be very difficult between syndromes because of their phenotypic variability. Attenuated FAP, MAP and Lynch syndrome could be all associated with fewer numbers of adenomas (3 - 10 polyps), nevertheless, each syndrome has distinct cancer risks, characteristic clinical features, and separate genetic etiologies. Thus, differential diagnosis is essential for correct management of the specific disease. In our laboratory we set up a methodology for genetic tests of the colorectal polyposis syndrome. In these reviews we summarize the literature data and our experience about diagnosis, genetic tests and cancer risk assesment associated with colorectal polyposis. According to literature data, in our

${ }^{*}$ Corresponding author. experience, there is a portion of analyzing patients that remain without identified mutation, after molecular screening of the specific gene involved in the pathogenesis of the disease. Since the sensibility of used techniques, such as DHPLC, MLPA and sequencing, is now very high, we suggest that a different approach to molecular diagnosis of polyposis syndromes is necessary. In our laboratory, we are now planning to set up analysis of a larger pannel of genes that could be involved in colorectal poliposis syndromes, using a next generation sequencing techniques. In our opinion, a better characterization of molecular basis of the polyposis syndromes will allow a more efficient cancer prevention.

Keywords: Familial Adenomatous Polyposis (FAP); MUTYH-Associated Polyposis (MAP); Peutz-Jeghers Syndrome (PJS); PTEN Hamartoma Tumour Syndrome (PHTS)

\section{FAMILIAL POLYPOSIS SYNDROMES INTRODUCTION}

For familial hereditary polyposis the differential diagnosis includes FAP, AFAP, MAP and the hamartomatous polyposis syndromes $[1,2]$.

\section{FAMILIAL ADENOMATOUS POLYPOSIS}

\subsection{Clinical Description}

Familial adenomatous polyposis (FAP) is a rare autosomal dominantly inherited disease, associated with mutations in the adenomatous polyposis coli (APC; MIM\# 175100) gene located at chromosome 5q21. The incidence for FAP is estimated at about 1 in 10,000 individuals and it accounts for about $1 \%$ of all colorectal cancers 
[3]. Affected individuals develop hundred to thousands of adenomatous polyps at a young age (second decade of life) that, if untreated, inevitably progress to colorectal carcinoma. Consequently, a genetic test is crucial 1) to identify mutation-carrying relatives for preventive measures, and 2) to reassure relatives who are free from gene mutations [1,2]. Although, APC germline disease-causing mutations have a penetrance close to $100 \%$, recent evidence points to very close genotype/phenotype relationships and a marked variation in the phenotypic expression of FAP. Severe phenotypes, characterized by more than 5000 polyps and early onset of the disease, are associated with mutations between codons 1250 and 1464, whereas patients with classical FAP have hundreds to thousands of adenomatous polyps in the colorectum during the second and third decades of life [4]. Germline mutations between codons 168 and 1680 and whole APC deletions are associated with classical phenotypes. Attenuated FAP, showing few polyps (about $10-100$ ), are caused by mutations at the extreme 5 ' or 3 ' ends of the APC gene or it could give rise to mutations in alternatively spliced exon [5]. Phenotypic variation of FAP, genetically indistinguishable, are Turcot syndrome (colorectal multiple adenomas associated with brain tumours) and Gardner syndrome (colorectal multiple adenomas with epidermoid cysts, osteomas, dental anomalies, and desmoid tumours) [1].

\subsection{Extra Colonic Manifestations}

FAP patients may also develop extracolonic manifestations. Both FAP and AFAP often arise with polyps in the upper gastrointestinal tract. About $50 \%$ of affected individuals develop profuse polyposis of gastric fundic gland and/or adenomatous polyps in the stomach [1]. Adenomatous polyps of the duodenum are observed in more than $50 \%$ of individuals and are commonly found in the second and third portions [6]. However, the most frequent extra colonic manifestations are congenital hypertrophy of the retinal pigment epithelium (CHRPE), which is present in about $80 \%$ of cases, and desmoid tumours, which are seen in about $13 \%$ of FAP families, often recurring in the mesentery or in the abdominal wall after resection. A papillary thyroid carcinoma and heaptoblastoma in the children of people with FAP have also been reported [7].

The lifetime cancer risk of FAP patients is about $1 \%$ of gastric cancer [8], 4\% - 12\% for duodenal cancer and about $10 \%$ of thyroid cancer. They also present elevated risk to develop stomach, liver (hepatoblastoma) and CNS (medulloblastoma) cancer. Nonmalignant lesion also occurs including osteomas (of the skull and mandible), epidermoid cysts, fibromas and dental abnormalities (unerupted teeth or congenital absence of teeth). These find- ings are less common in attenuated FAP [1].

\subsection{Genetics}

FAP and attenuated FAP are caused by germline mutations in the APC gene and are inherited in an autosomal dominant manner, so the children of a subject affected by FAP have a $50 \%$ risk of developing the disease. De novo mutations are responsible for approximately $25 \%$ of FAP cases [9]. In $10 \%$ to $15 \%$ of de novo mutations a somatic APC mosaicism may be present [10]. APC is an ubiquitously expressed tumour suppressor gene located on the long arm of chromosome 5 at position $5 \mathrm{q} 21$. It contains at least 21 exons, 17 of which are coding exons and 4 are untranslated exons. The APC gene encodes a large protein of 309 kilo-Daltons containing multiple domains [3]. By interacting with several cellular proteins, it is mainly involved in Wnt signaling, in part by regulating $\beta$-catenin levels, but also in intercellular adhesion, cytoskeleton stabilization, signal transduction, apoptosis and cell-cycle control, and it probably exerts a nuclear function in chromosome segregation.

APC binds to $\beta$-catenin inducing ubiquitin-mediated $\beta$-catenin degradation and, in this way, it negatively regulates transcription of $\beta$-catenin targets [11]. Its functional domains include heptad repeats at the amino-terminal end (amino acids 6 - 57) that mediate homodimer formation, the armadillo repeat (amino acids 453 - 767) that binds to Asef, two motifs that interact with $\beta$-catenin at amino acids 1020 - 1169 and 1262 - 2033, respectively, and microtubule-, EB1- and hDLG-binding domains.

\subsection{Genotype-Phenotype Correlations}

Although, APC germline disease-causing mutations have a penetrance close to $100 \%$, recent evidence points to the very close genotype/phenotype relationships and the clinical manifestations and severity of FAP vary greatly with the mutation site [1]. The disorder is classically characterized by more than 100 colorectal adenomas, early onset of colorectal carcinoma, and specific extracolonic features. Attenuated Familial adenomatous polyposis (AF$\mathrm{AP})$ is a milder form of the disease in which patients have less than 100 adenomas. AFAP patients with dominant inheritance harbor germ-line mutations in the 5' or 3' regions of the APC gene, to codon 1517 or distal to codon 1900, or in regions affected by alternative splicing events. Severe phenotypes, characterized by more than 5000 polyps and early onset of the disease, are associated with mutations between codons 1250 and 1464, whereas patients with classical FAP have hundreds to thousands of adenomatous polyps in the colorectum during the second and third decades of life [4,12]. Mutations contributing to classical FAP occur between exon 5 and the 5' portion of exon 15 [11]. CHRPE is associated with muta- 
tions between codons 457 and 1444 [13]. Desmoid tumours, found in approximately $10 \%$ of FAP patients, are present in patients with mutations between codons 1403 and 1578. Although rare, FAP patients have an increased risk of hepatoblastoma associated with mutations between codons 457 and 1309 [14].

\section{MUTYH-ASSOCIATED POLYPOSIS (MAP)}

\subsection{Clinical Description}

Another group of patients showing recessive inheritance and FAP or AFAP phenotype often tests negative for APC mutations but harbor inherited biallelic mutations in the base-excision repair (BER) gene MYH (MUTYH; OMIM\# 604933, Gene Bank NM_012222.1). MUTYH (MYH) gene is localized on chromosome 1 at position $1 \mathrm{p}$ and acts together with MTH1 and OGG1 as part of the base excision repair machinery $[15,16]$. MAP patients develop adenomatous polyposis of the colon-rectum and show an increased risk of CRC. Although the colonic phenotype of MAP is of attenuated FAP, biallelic MUTYH mutations have also been associated with early onset CRC and few or no polyps. Hyperplastic polyps are present, even though adenomatous polyps are more largely represented [1]. FAP-associated extra-colonic manifestations such as osteomas, desmoids, CHRPE and thyroid cancer did not take place but a higher risk of ovarian, bladder, skin, sebaceous gland tumors and breast cancer was described [17].

\subsection{Genetics}

MAP accounts for about $18 \%$ of the APC-negative AFAP families, is caused by MUTYH mutations. It is inherited in a recessive manner and family history is less informative in the diagnosis of this syndrome. The MUTYH is a base excision repair protein that provides defence against oxidation-induced DNA damage by removing A mispaired with 8-oxo-7, 8-dihydro2'deoxyguanosine (8oxoG), the most stable deleterious products of oxidative DNA damage. Hence, it prevents $\mathrm{G}: \mathrm{C}$ to $\mathrm{T}: \mathrm{A}$ transversions caused by oxidative stress. Genetic testing for MAP is suggested in AFAP individuals negative for APC mutation. Identification and characterization of the specific MUTYH mutation confirm the diagnosis and allows genetic testing in family members. Biallelic MYH mutations are associated with adenomatous colorectal polyps and a very high risk of CRC. Nevertheless, monoallelic MUTYH mutations might act as low-penetrance CRC susceptibility modifiers and/or cooperate with other gene mutations. Parents and children of individuals with MAP are rarely affected and siblings of individuals with biallelic MUTYH mutations have a $25 \%$ possibility to be affected and they should be informed about the risks [2]. The obligate heterozygote children of MAP patient and a nonconsanguineous healthy partner have only a small risk of developing MAP (about 1\%). The CRC risk of heterozygous carriers is still controversial. Recent systematic studies in large patient populations estimate the relative risk at 1.5 to 2.1 in relation to the general population [9].

\subsection{Mutation-Negative Adenomatous Polyposis}

In about $20 \%$ of families with FAP and in $70 \%$ of AFAP families the cause of adenomatous polyposis remains unknown and the specific pathogenic mutations have not been identified. The absence of detectable mutations could be explained by misdiagnoses, the existence of an alternative gene inactivation mechanism that remains undiscovered by routine screening methods or, alternatively, by genetic heterogeneity. A monogenic or multifactorial etiology is a possible explanation. In patients showing attenuate FAP phenotype without extracolonic manifestations and with uninformative family history, HNPCC or other polyposis syndromes should be considered in the differential diagnosis. On the other hand, an individualized approach may often be necessary because mutations in APC or MYH are sometimes associated with variable phenotype and mutations have been reported in APC causing an increment of CRC risk but not adenomatous colorectal polyposis [9].

\subsection{Phenotypic Heterogeneity of FAP Patients from Southern Italy}

Our knowledge points to various genotype/phenotype correlations within familial adenomatous polyposis patients. All patients with a totally deleted APC gene and most patients with point mutations within exon 15 had a classical FAP phenotype with CHRPE [18]. We described three families carrier of the full APC gene deletion, one of whom showing intrafamilial phenotypic variability. Among affected subjects colonic disease manifestations were expressed at considerably different ages and the number of polyps ranged from few in some patients to hundreds of others; extracolonic manifestations, i.e. kidney carcinoma and calcifying epithelioma, were present in one of these patients. These data support the notion that other genetic and/or environmental factors could play an important role in the penetrance of the disease [19]. Furthermore, in our study population, very severe polyposis is associated with mutations that map in a region comprised from codon 1249 to codon 1330, but phenotypic variability is often described. We reported a severe case of FAP in a young child with very early onset of the disease. A 10-year-old female was evaluated 
for anemia and an 8-year-long history of recurrent hematochezia (initial disease presentation at the age of 2 years). Family history was negative for FAP, other polyposis syndromes, or colon cancer. Molecular diagnosis performed in this proband revealed the presence of 1309 APC gene deletion that was not found in the proband's relatives and therefore represented a de novo mutation. This mutation consists in a deletion of AAAGA bases from sites 3927 to 3931 of the APC gene and it has been found in about twenty percent of all FAP pedigrees typically associated with severe, early-onset disease and with congenital hypertrophy of the retinal pigment epithelium (CHRPE). This mutation is responsible for the rare cases of polyposis that occur in the first decade of life. The proband presented very early onset of disease although she did not show a family history of FAP. Therefore, it is important that a child with irondeficiency anemia and a long history of rectal bleeding has an early and adequate endoscopic investigation [20]. In another family we described a mutation identified in exon 5 (595_596insG), a region usually mutated in attenuated polyposis, associated with a particularly severe phenotype, without $\mathrm{CH}-$ RPE [21]. Patient bearing this mutation was also affected by desmoid tumours, mandibular osteomas, and tubular adenomas with moderate dysplasia in the colon. Interestingly, a patient bearing a different mutation in this region (591_592delAG) showed a classical pheno-type.

Two other families, bearing mutations $4621 \mathrm{C}>\mathrm{T}$ (Q1541X) and 4526_27insT, respectively, had similar extracolonic manifestations, i.e. fundic gland polyps, which are the most common gastric polyps, and cranial osteomas. However, the patient with mutation $4621 \mathrm{C}>\mathrm{T}$ (Q1541X) had a classical FAP phenotype associated with abdominal desmoid tumours, whereas the patient with mutation 4526_27insT had attenuated polyposis with few adenomatous polyps (about 10) in the colon, in contrast to the genotype/phenotype correlation usually reported for this mutation site. This finding lends weight to the intra- and inter-familial variability observed in FAP families [18].

Alterations of APC mRNA were also identified as pathogenic for a subset of FAP cases in which no germline APC or MYH mutations have been detected. About $15 \%$ of inherited human diseases involve splicing errors caused by mutations in splice sites or in splicing control sequences. We described a 62-year-old man without a dominant family history of FAP and an attenuated FAP phenotype with fewer colonic polyps (about 10). The family history was unclear; in fact, a cousin died of colorectal cancer at the age of 65 years, but his parents died from causes other than colorectal cancer. This FAP patient had a missense mutation in exon 15, c.4909G- > A (p.D1637N), which consists in a G to A transition at po- sition 4909 and causes a substitution of the aspargine 1637 to aspartic acid. No other mutation was found.

Using the "ESEfinder" program, binding motifs in known splicing enhancer proteins (SR proteins: SF2/ASF, SC35, SRp40, and SRp55) were found in this region, in both normal and mutated sequences. Remarkably, we identified an increase of the SC35 binding motif affinity in the mutated sequence (score 3.0) compared to the control sequence (score 2.5). An unusual feature of mutation $4909 \mathrm{G}->\mathrm{A}$ is that the change does not alter an authentic splice site nor does it generate a cryptic splice site, rather, the mutation probably activates an ESE control sequence that indirectly activates a cryptic splice junction upstream of the mutated site within the same exon.

Moreover, our study on an Italian FAP population confirm that MYH associated polyposis (MAP) genotype-phenotype correlations are very complex. Biallelic MYH mutations can result in either classic or attenuated polyposis, furthermore, patients with the same MYH biallelic mutations can show different phenotypes. Finally, in a relevant fraction of patients with colon polyposis, a mutation was found in a single MYH allele (monoallelic mutation) [22].

\section{HAMARTOMATOUS POLYPOSIS SYNDROMES}

The hamartomatous polyposis syndromes are a heterogeneous group of disorders including less than $1 \%$ of all hereditary colorectal cancers. They are characterized by autosomal-dominant inheritance, development of gastrointestinal hamartomatous polyps and are associated with GI and extraintestinal malignancy $[23,24]$. The hamartomatous polyposis syndromes include Peutz-Jeghers syndrome (PJS); PTEN hamartoma tumour syndrome (PHTS), comprehending Cowden syndrome (CS) and Bannayan-Riley-Ruvalcaba syndrome (BRRS); and juvenile polyposis syndrome (JPS). Differential diagnosis are essential for suitable patient management, because each of these syndromes presents its own characteristic organspecific manifestations and each requires a precise surveillance approach [25].

\subsection{Peutz-Jeghers Syndrome: Clinical Description, Extraintestinal Manifestations and Genetics}

Peutz-Jeghers syndrome (PJS) is an autosomal dominantly inherited syndrome characterized by mucocutanoeus pigmentation, multiple hamartomatous polyps in the gastrointestinal tract and an increased risk of cancer at a young age. It is the most common form of hamartomatous polyposis, with a reported prevalence of between 1 in 29,000 and 1 in 200,000 [26,27]. Inactivating 
germ-line mutations in the tumour suppressor gene STK11/LKB1 have been detected in approximately $80 \%$ of patients [2]. The polyps, commonly found in the small intestine but also in the stomach and colon, have a muscular core, are histologically classified as hamartomas and their numbers may range from 1 to a complete carpeting of the gastrointestinal tract [2]. Abdominal pain, intussusception, anemia, melena, hematochezia, hematemesis, and obstruction are the main PJS clinical manifestations. Pigmentation is seen around the lips (in over $95 \%$ of cases), in the buccal mucosa (about $80 \%$ of cases), on the hands, feet, genitals, and around the nose and eyes. Pigmentation is typically present in early childhood and starts to become dim usually after the start of puberty [25]. Approximately one third of PJS patients present a disease onset in the first decade of life, while up to $60 \%$ by the second or third decade [28].

Extraintestinal polyps have been reported in the nose, bronchi, renal pelvis, bladder, in the gallbladder and bile ducts. PJS is also associated with various malignancies. In addition to an elevated risk of gastrointestinal malignnancies, such as gastroesophageal, small bowel and colorectal cancer, an increased risk of cancer at other sites, particularly in breast, pancreas, ovary, uterus, cervix, lung and testicle, have been reported. The extimated risk of cancer is of $60 \%$ by 60 years of age and $85 \%$ by 70 years of age. The risk of developing cancer by 60 years of age was estimated at about $31 \%$ for gastrointestinal and breast cancer, $18 \%$ for gynecologic cancer, $7 \%$ for pancreatic cancer and $13 \%$ for lung cancer [28].

Sex cord tumours and Sertoli cell tumours, both arising from the same embryonic tissue, are often described in women and men affected by PJS [25].

The gene associated with PJS is a serine-threonine kinase that is located on chromosome 19p13.3. Germline mutations in LKB1/STK11 gene has been reported in $80 \%$ of patients with PJS. In the remaining $20 \%$ of PJS patients, defects in other genes or not yet identified ways of LKB1 inactivation might be responsible for PJS. Several putative candidate genes have been studied, including genes encoding LKB1 interacting proteins; so far a second PJS gene has not been identified. Common mutations are frameshift and nonsense mutations in exons 1 - 6; however, large deletion mutations missed by direct sequencing have been recently described using multiple ligation probes, long range RCR and Real time PCR [2830]. LKB1 forms a complex with pseudokinase STRAD and the scaffolding protein $\mathrm{MO} 25$, that activates at least 14 serine/threonine kinase by phosphorylation of the "T-Loop" threonine localized in their kinase domain. This implicates the involvement of LKB1 in several signalling pathways. The first identified physiological substrate of LKB1 was AMPK (AMP-activated protein kinase), which is a master regulator of cellular energy charge. In addition to the involvement in energy metabolism, LKB1 has the capacity to regulate multiple cellular processes, such as cell cycle arrest, Wnt signalling, cell polarity, transforming growth factor beta signalling, p53dependent apoptosis and chromatin remodelling [31].

We examined a population of 10 Italian PJS patiens from Southern Italy, seting up a strategy to screen the entire coding region of the LKB1 gene, both at DNA and RNA level. We described an intra-exonic in-frame deletion encompassing exons 2 and 4 and characterized the breakpoints of this LKB1/STK11 intragenic deletion. This rearrangement, that deletes about $7 \mathrm{~kb}$ of the LKB1 genomic region encompassing exons 2 and 3 is most likely an Alu-Alu homologous recombination event. Two 26 bp core sequences of two Alu elements (both AluY sequences), showing a $96 \%$ homology, are indeed localized at the 5' and 3' end of the breakpoints, respectively. This sequence, could itself act as a recombinase. Alumediated homologous recombination is a mechanism well documented so far, however it was the first evidence that this mechanism is involved in the STK11/ LKB1 gene rearrangements. According to other literature data, we identified the disease causing mutations in about $67 \%$ of PJS patients, suggesting that others gene inactivating mechanisms might be responsible for PJS in our mutation negative population subset [29].

\subsection{PTEN Hamartoma Tumour Syndrome (PHTS)}

The "PTEN hamartoma tumour syndrome" (PHTS) is inherited in an autosomal dominant manner and includes a group of rare multiple hamartoma syndromes, such as Cowden syndrome (CS), Bannayan-Riley-Ruvalcaba syndrome (BRRS), Proteus syndrome (PS), and Proteus-like syndrome, that are caused by germline mutations within the tumour suppressor gene "phosphatase and tensin homolog deleted on chromosome ten" (PTEN). These syndromes are characterized by multiple amartomatous polyps in the gastrointestinal tract and by a greatly increased risk of developing malignant tumours in many tissues. Because of many of its phenotypic manifestations are subtle and occur in the general population, its phenotypic variability and incomplete penetrance, PHTS is likely to be underdiagnosed [32].

\subsection{Cowden Syndrome}

CS is a rare autosomal dominant syndrome that occur in 1 in 200,000 individuals. This syndrome is characterized by macrocephaly, mucocutaneous lesions (such as facial trichilemmoma), acral keratosis, papillomatous papules and glycogenic acanthosis of the esophagus, which involves large benign glycogen-filled epithelial cells that are gray to white in color. It is also associated with thy- 
roid, breast, and endometrial manifestations, including cancer in all of these areas. Renal cancer has also been associated with CS; the risk of developing gastrointestinal carcinoma in CS is still unclear. The incidence of gastrointestinal polyps in CS differs in the literature, varying from $30 \%$ to $85 \%$, reporting an incidence of gastrointestinal polyps in CS less than in BRRS [25]. The specific pathogenic PTEN gene mutations are identified in approximately $85 \%$ of individuals who meet the diagnostic criteria for CS and in about $65 \%$ of individuals with a clinical diagnosis of BRRS. The most important aspect of the management of an individual carrier of a PTEN mutation is increased cancer surveillance because of the most serious consequences of PHTS consist in the increased risk of breast, thyroid, endometrial, and renal cancer [31,32].

\subsection{Bannayan-Riley-Ruvalcaba Syndrome}

Clinical Presentation: The BRRS is a congenital disorder characterized by macrocephaly, intestinal hamartomatous polyposis, lipomas, and pigmented macules of the glans penis. It is suggested that individuals with BRRS should be considered at risk for malignancy, as with CS.

The incidence of gastrointestinal polyps in BRRS has been reported to be $45 \%$. The extraintestinal manifestations, consisting of macrocephaly, developmental delay, accelerated growth of first metacarpal and first proximal and middle phalanges, joint hyperflexibility, pectus excavatum, scoliosis, genital pigmentation, lipomas, hemangiomas, lipid storage myopathy, are the hallmarks of this syndrome [33-36].

\subsection{Cowden Syndrome and Bannayan-Riley-Ruvalcaba Syndrome Genetics}

CS and BRRS are allelic diseases with an autosomal dominant inheritance pattern and variable penetrance, in which a mutation of the PTEN gene, located on chromosome $10 \mathrm{q} 22-23$, is found in $80 \%$ of CS patients and $60 \%$ of BRRS patients $[37,38]$. Close to 100 different germline mutations of PTEN have been reported to date encompassing point, nonsense, frame shift, splice site, missense, and deletion/insertion mutations. Most mutations occur in exon 5 , but mutations in all other exons, except the first, have also been described. Around $10 \%$ of PTEN mutations occur in the promoter region and the role of epigenetic regulation is not well outlined.

Balanced translocations and deletions and mutations in exons 6 and 7 occur preferentially in BRRS, while mutations in all exons except 1,4 , and 9 have been found in CS. Recently, differential expression of the PTEN gene has been correlated with the different phenotypes of CS and BRRS.
PTEN is a 9-exon tumour suppressor gene that encodes for a 403 amino acid tyrosine phosphatase protein that dephosphorylates tyrosine, serine, and threonine. It acts as a lipid phosphatase to negatively regulate the PI3K/AKT/mTOR pathway [39]. Recently, nuclear compartmentalization of PTEN has been found as a key component of its tumour-suppressive activity. PTEN is also involved in regulating the cell cycle, apoptosis, and angiogenesis. Evidence exists indicating that PTEN is a functionally haploinsufficient tumour suppressor gene.

A new study revealed that about $10 \%$ of CS and CSL individuals, negative for germline PTEN mutations, harbor germline variants in SDHB and SDHD. These mutations are associated with a greater risk of developing breast, thyroid, and renal cell carcinomas consistent with germline PTEN mutations and SDHB mutations observations in pheochromocytoma/PGL patients, who were also found to have a risk of papillary thyroid cancer and early-onset renal cancers [40].

\subsection{An Overview of PHTS Patients from Southern Italy}

We performed mutational analysis of the PTEN gene in three PHTS patients, setting up a combination of RT$\mathrm{PCR}$ reaction of the whole cDNA, PCR of genomic region, including the promoter region from $b p-1398$ to $b p$ +1 , sequencing of the amplified fragments, Real Time PCR and western blot techniques. The first PHTS patient (PHTS1), affected by BRRS, had a missense mutation named c.406T $\rightarrow \mathrm{C}$ in exon 5 of the PTEN gene. This mutation, localized in the catalitic domain of PTEN protein, determines the aminoacidic change of cysteine residue 136 into an arginine. The other two patients, (both affected by $\mathrm{CS}$ ), showed a significant decrease in the PTEN mRNA expression when analysed by Real Time quantitative RT-PCR.

PI3K-Akt signalling activation is related to $\beta$-catenin phosphorylation at Ser 552 and its stabilization, nuclear accumulation and transcriptional activation. Furthermore, PI3K-Akt signalling activation results in the inactivation of GSK- $3 \beta$ and reduces N-terminal $\beta$-catenin phosphorylation, which is associated with its degradation.

Thus, we provided the first evidence of $\beta$-catenin accumulation in non-neoplastic cells of PHTS patients, caused by germ-line PTEN alteration without a "second hit" of gene inactivation taking place. In light of these data, we suggest that $\beta$-catenin could represent a good candidate as a diagnostic marker for hereditary colorectal diseases that determine $\beta$-catenin accumulation. This is mainly noteworthy for PHTS syndrome, which is often underdiagnosed. In addition, all PHTS patients analysed showed alterations in the expression of TNF $\alpha$, its receptors and IL-10 [41]. 


\section{THE DIFFERENTIAL DIAGNOSIS AND SURVEILLANCE OF HEREDITARY GASTROINTESTINAL POLYPOSIS SYNDROMES}

Differential diagnosis and identification of the polyposis syndromes is essential for management and cancer prevention for affected individuals, because of each polyposis syndrome has its own distinctive organ-specific manifestation and each requires a different surveillance strategy. Polyposis patients have a high lifetime risk of gastrointestinal and extraintestinal carcinoma and their first-degree relatives have a high risk of recurrence of the syndrome. Differential diagnosis and identification of the polyposis syndromes is essential for management and cancer prevention for affected individuals, because of each polyposis syndrome has its own distinctive organspecific manifestation and each requires a different surveillance strategy. Polyposis patients have a high lifetime risk of gastrointestinal and extraintestinal carcinoma and their first-degree relatives have a high risk of recurrence of the syndrome. Characterization of a causative mutation in leukocyte DNA is essential for the differenttial diagnosis among the various adenomatous polyposis syndromes, assessment of the risk of recurrence (autosomal dominant versus autosomal recessive inheritance), determination of familial cancer risks, based on genespecific cancer associations and predictive testing of asymptomatic at risk individuals. The role of molecular genetic findings in treatment decisions, on the other hand, is limited because identification of a germline mutation rarely allows any estimation of the likely course of the disease. According to international literature data, we suggest that next-generation sequencing is today the better and more efficient technique for molecular diagnosis of hereditary colorectal polyposis syndrome, hereditary colorectal cancer and familial colorectal cancer. Indeed, it allows to consider a number of different genes associated with colon cancer for differential diagnosis. Using this solid technique, we are going to analyze a gene panel including APC, MUTYH, PTEN, STK11, BMPR1A, SMAD4, MLH1, MSH2, MSH6, PMS2, CDH1, CHEK2, EPCAM and TP53. This approach could consent to detect previously unidentified low frequency allelic variants including a novel candidate locus. Moreover, even if no mutation is found, the patient with polyposis still needs to be treated appropriately and clinical follow up should be initiated even before mutation testing is complete [40].

\section{REFERENCES}

[1] Jasperson, K.W., Tuohy, T.M., Neklason, D.W. and Burt, R.W. (2010) Hereditary and familial colon cancer. Gastroenterology, 138, 2044-2058. doi:10.1053/j.gastro.2010.01.054

[2] Gallagher, D.J., Smith, J.D., Offit, K. and Stadler, Z.K. (2010) Diagnosing hereditary colorectal cancer. Clinical Colorectal Cancer, 9, 205-211. doi:10.3816/CCC.2010.n.030

[3] Groden, J., Thliveris, A., Samowitz, W., Carlson, M., Gelbert, L., Albertsen, H., et al. (1991) Identification and characterization of the familial adenomatous polyposis coli gene. Cell, 66, 589-600. doi:10.1016/0092-8674(81)90021-0

[4] Ritchie, K.J., Walsh, S., Sansom, O.J., Henderson, C.J. and Wolf, C.R. (2009) Markedly enhanced colon tumourigenesis in ApcMin mice lacking glutathione Stransferase Pi. Proceedings of the National Academy of Sciences of the United States of America, 8, 20859-20864.

[5] Burt, R.W., Leppert, M.F., Slattery, M.L., Samowitz, W.S., Spirio, L.N., Kerber, R.A., Kuwada, S.K., Neklason, D.W., Disario, J.A., Lyon, E., Hughes, J.P., Chey, W.Y. and White, R.L. (2004) Genetic testing and phenotype in a large kindred with attenuated familial adenomatous polyposis. Gastroenterology, 127, 444-451. doi:10.1053/i.gastro.2004.05.003

[6] Bülow, S., Björk, J., Christensen, I.J., Fausa, O., Järvinen, H., Moesgaard, F., et al. (2004) Duodenal adenomatosis in familial adenomatous polyposis. Gut, 53, 381-386. doi:10.1136/gut.2003.027771

[7] Djureinovic, T., Skoglund, J., Vandrovcova, J., Zhou, X.L., Kalushkova, A., Iselius, L. and Lindblom, A. (2006) A genome wide linkage analysis in Swedish families with hereditary nonfamilial adenomatous polyposis/non-hereditary non-polyposis colorectal cancer. Gut, 55, 362-366. doi:10.1136/gut.2005.075333

[8] Bianchi, L.K., Burke, C.A., Bennett, A.E., Lopez, R., Hasson, H. and Church, J.M. (2008) Fundic gland polyp dysplasia is common in familial adenomatous polyposis. Clinical Gastroenterology and Hepatology, 6, 180-185. doi:10.1016/j.cgh.2007.11.018

[9] Aretz, S. (2010) The differential diagnosis and surveillance of hereditary gastrointestinal polyposis syndromes. Deutsches Ärzteblatt International, 107, 163-173.

[10] Aretz, S., Stienen, D., Friedrichs, N., Stemmler, S., Uhlhaas, S., Rahner, N., Propping, P. and Friedl, W. (2007) Somatic APC mosaicism: A frequent cause of familial adenomatous polyposis (FAP). Human Mutation, 28, $985-$ 992. doi:10.1002/humu.20549

[11] Half, E., Bercovich, D. and Rozen, P. (2009) Familial adenomatous polyposis. Orphanet Journal of Rare Diseases, 12, 4-22.

[12] Nieuwenhuis, M.H. and Vasen, H.F. (2007) Correlations between mutation site in APC and phenotype of familial adenomatous polyposis (FAP): A review of the literature. Critical Reviews in Oncology/Hematology, 61, 153-161. doi:10.1016/j.critrevonc.2006.07.004

[13] Wallis, Y.L., Macdonald, F., Hultén, M., Morton, J.E.V., McKeown, C.M., Neoptolemos, J.P., Keighley, M. and Dion, G. (1994) Genotypephenotype correlation between position of constitutional APC gene mutation and CHRPE expression in FAP. Human Genetics, 5, 543-548. 
[14] Bisgaard, M.L. and Bulow, S. (2006) Familial adenomatous polyposis (FAP): Genotype correlation to FAP phenotype with osteomas and sebaceous cysts. American Journal of Medical Genetics, 140, 200-204.

[15] Sieber, O.M., Lipton, L., Crabtree, M., Heinimann, K., Fidalgo, P., Phillips, R.K., et al. (2003) Multiple colorectal adenomas, classic adenomatous polyposis, and germline mutations in MYH. New England Journal of Medicine, 348, 791-799. doi:10.1056/NEJMoa025283

[16] Al-Tassan, N., Chmiel, N.H., Maynard, J., Fleming, N., Livingston, A.L., Williams, G.T., et al. (2002) Inherited variants of $\mathrm{MYH}$ associated with somatic $\mathrm{G}: \mathrm{C} \rightarrow \mathrm{T}: \mathrm{A}$ mutations in colorectal tumors. Nature Genetics, 30, 227232. doi: $10.1038 / \mathrm{ng} 828$

[17] Vogt, S., Jones, N., Christian, D., Engel, C., Nielsen, M., Kaufmann, A., Steinke, V., Vasen, H.F., Propping, P., Sampson, J.R., Hes, F.J. and Aretz, S. (2009) Expanded extracolonic tumour spectrum in MUTYH-associated polyposis. Gastroenterology, 137, 1976-1985. doi:10.1053/i.gastro.2009.08.052

[18] De Rosa, M., Scarano, M.I., Panariello, L., Morelli, G., Riegler, G., Rossi, G.B., Tempesta, A., Romano, G., Renda, A., Pettinato, G. and Izzo P. (2003) The mutation spectrum of the APC gene in FAP patients from Southern Italy: Detection of known and four novel mutations. $\mathrm{Hu}$ man Mutation, 21, 655-656. doi:10.1002/humu.9151

[19] De Rosa, M., Scarano, M.I., Panariello, L., Carlomagno, N., Rossi, G.B., Tempesta, A., Borgheresi, P., Renda, A. and Izzo, P. (1999) Three submicroscopic deletions at the APC locus and their rapid detection by quantitative-PCR analysis. European Journal of Human Genetics, 7, 695703. doi:10.1038/sj.ejhg. 5200344

[20] Auricchio, R., De Rosa, M., Quaglietta, L., Miele, E., Boccia, G., Staiano, A. and Izzo P. (2005) A dramatic case of early-onset familial adenomatous polyposis. Clinical Genetics, 67, 104-106. doi:10.1111/j.1399-0004.2005.00381.x

[21] Scarano, M.I., De Rosa, M., Gentile, M., Bucci, L., Ferulano, G.P., Carlomagno, N., Renda, A., Guanti, G., Salvatore, F. and Izzo, P. (1997) Three novel germline mutations in the adenomatous polyposis coli gene. Human Mutation, 9, 191-193. doi:10.1002/(SICI)1098-1004(1997)9:2<191::AID-HUM U16>3.0.CO;2-Y

[22] De Rosa, M., Galatola, M., Borriello, S., Duraturo, F., Masone, S. and Izzo, P. (2009) Implication of adenomatous polyposis coli and MUTYH mutations in familial colorectal polyposis. Diseases of the Colon \& Rectum, 52, 268-274. doi:10.1007/DCR.0b013e318197d15c

[23] Attard, T.M. and Young, R.J. (2006) Diagnosis and management of gastrointestinal polyps: Pediatric considerations. Gastroenterology Nursing, 29, 16-22. doi:10.1097/00001610-200601000-00003

[24] Wirtzfeld, D.A., Petrelli, N.J. and Rodriguez-Bigas, M.A. (2001) Hamartomatous polyposis syndromes: Molecular genetics, neoplastic risk, and surveillance recommendations. Annals of Surgical Oncology, 8, 319-327. doi:10.1007/s10434-001-0319-7

[25] Manfredi, M. (2010) Hereditary hamartomatous poly- posis syndromes: understanding the disease risks as children reach adulthood. Gastroenterology \& Hepatology, 6, 185-196.

[26] Giardiello, F.M. and Trimbath, J.D. (2006) Peutz-Jeghers syndrome and management recommendations. Clinical Gastroenterology and Hepatology, 4, 408-415. doi:10.1016/j.cgh.2005.11.005

[27] Jeghers, H., McKusick, V.A. and Katz, K.H. (1949) Generalized intestinal polyposis and melanin spots of the oral mucosa, lips and digits: A syndrome of diagnostic significance. New England Journal of Medicine, 241, 10311036. doi:10.1056/NEJM194912292412601

[28] Hearle, N., Schumacher, V., Menko, F.H., Olschwang, S., Boardman, L.A., Gille, J.J., et al. (2006) Frequency and spectrum of cancers in the Peutz-Jeghers syndrome. Clinical Cancer Research, 12, 3209-3215. doi:10.1158/1078-0432.CCR-06-0083

[29] De Rosa, M., Galatola, M., Quaglietta, L., Miele, E., De Palma, G., Rossi, G.B., Staiano, A. and Izzo, P. (2010) Alu-mediated genomic deletion of the serine/threonine protein kinase 11 (STK11) gene in Peutz-Jeghers syndrome. Gastroenterology, 138, 2558-2560. doi:10.1053/j.gastro.2010.03.061

[30] Marignani, P.A. (2005) LKB1, the multitasking tumour suppressor kinase. Journal of Clinical Pathology, 58, 1519. doi:10.1136/jcp.2003.015255

[31] Farooq, A., Walker, L.J., Bowling, J. and Audisio, R.A. (2010) Cowden syndrome. Cancer Treatment Reviews, 36, 577-583. doi:10.1016/j.ctrv.2010.04.002

[32] Eng, C. (2003) PTEN: One gene, many syndromes. $\mathrm{Hu}$ man Mutation, 22, 183-198.

[33] Gorlin, R.J., Cohen Jr., M.M., Condon, L.M. and Burke, B.A. (1992) Bannayan-Riley-Ruvalcaba syndrome. American Journal of Medical Genetics, 44, 307-314. doi:10.1002/ajmg.1320440309

[34] Ruvalcaba, R.H., Myhre, S. and Smith, D.W. (1980) Sotos syndrome with intestinal polyposis and pigmentary changes of the genitalia. Clinical Genetics, 18, 413-416. doi:10.1111/j.1399-0004.1980.tb01785.x

[35] Liaw, D., Marsh, D.J., Li, J., Dahia, P.L., Wang, S.I., Zheng, Z., et al. (1997) Germline mutations of the PTEN gene in Cowden disease, an inherited breast and thyroid cancer syndrome. Nature Genetics, 16, 64-67. doi:10.1038/ng0597-64

[36] Tan, M.H., Mester, J.L., Ngeow, J., Rybicki, L.A., Orloff, M.S. and Eng, C. (2012) Lifetime cancer risks in individuals with germline PTEN mutations. Clinical Cancer Research, 18, 400-407. doi:10.1158/1078-0432.CCR-11-2283

[37] Marsh, D.J., Kum, J.B., Lunetta, K.L., Bennett, M.J., Gorlin, R.J., Ahmed, S.F., et al. (1999) PTEN mutation spectrum and genotype-phenotype correlations in Bannayan-Riley-Ruvalcaba syndrome suggest a single entity with Cowden syndrome. Human Molecular Genetics, 8, 1461-1472. doi:10.1093/hmg/8.8.1461

[38] Marsh, D.J., Coulon, V., Lunetta, K.L., Rocca-Serra, P., Dahia, P.L., Zheng, Z., et al. (1998) Mutation spectrum and genotype-phenotype analyses in Cowden disease and 
Bannayan-Zonana syndrome, two hamartoma syndromes with germline PTEN mutation. Human Molecular Genetics, 7, 507-515. doi: $10.1093 / \mathrm{hmg} / 7.3 .507$

[39] Waite, K.A. and Eng, C. (2002) Protean PTEN: Form and function. American Journal of Human Genetics, 70, 829844. doi: $10.1086 / 340026$

[40] Eng, C. (2010) Mendelian genetics of rare-and not so rare-cancers. Annals of the New York Academy of Sci- ences, 1214, 70-82.

doi:10.1111/j.1749-6632.2010.05789.x

[41] Galatola, M., Paparo, L., Duraturo, F., Turano, M., Rossi, G.B., Izzo, P. and De Rosa, M. (2012) Beta catenin and cytokine pathway dysregulation in patients with manifestations of the "PTEN hamartoma tumour syndrome". BMC Medical Genetics, 13, 28. doi:10.1186/1471-2350-13-28 